\title{
ABPA diagnosis in cystic fibrosis patients: the clinical utility of IgE specific to recombinant Aspergillus fumigatus allergens
}

\author{
Marina B. Almeida, ${ }^{1}$ Maria Helena C. F. Bussamra, ${ }^{2}$ Joaquim C. Rodrigues $^{2}$
}

\begin{abstract}
Objective: Allergic bronchopulmonary aspergillosis (ABPA) is a complicating factor of cystic fibrosis which can result in a devastating combination as lung disease progresses. The overlap between the signs and symptoms of the two conditions makes diagnosis problematic, even if standardized criteria are used. The objective of this study was to identify, in a group of cystic fibrosis patients, cases of ABPA by assaying IgE specific to recombinant Aspergillus fumigatus antigens and to compare the method with the Cystic Fibrosis Foundation diagnostic criteria.

Methods: Fifty-four patients, aged 2 to 20 years, presenting characteristics that could occur with ABPA in isolation, were systematically assessed based on the following: clinical data, a chest CT scan, immediate hypersensitivity skin test for $A$. fumigatus, total serum IgE assay, RAST for $A$. fumigatus and serum IgE specific for the recombinant allergens Asp f1, f2, f3, f4 and f6.

Results: Thirty-nine patients were eligible for the study. Thirty-two of these were investigated. Sensitization to $A$. fumigatus was observed in $34 \%$. Both the Cystic Fibrosis Foundation criteria and the recombinant antigen specific IgE assay defined three patients as suffering from ABPA; however, only two of these patients were diagnosed by both methods.

Conclusions: The detection of $A$. fumigatus recombinant antigen specific IgE was a useful tool for the early detection of sensitization and diagnosis of ABPA. Nevertheless, diagnostic confirmation cannot be divorced from clinical findings, and before this method can be used for ABPA diagnosis, for detecting relapses and for defining cure criteria, longitudinal studies with larger numbers of patients are required.
\end{abstract}

J Pediatr (Rio J). 2006;82(3):215-20: Allergic bronchopulmonary aspergillosis, Aspergillus fumigatus, cystic fibrosis, antigens.

\section{Introduction}

Allergic bronchopulmonary aspergillosis (ABPA) is a lung hypersensitivity disease mediated by an allergic late-phase inflammatory response to certain antigens of Aspergillus fumigatus. ${ }^{1}$ In patients with cystic fibrosis $(\mathrm{CF})$, the presence of atopic disease, bronchial hypersecretion and a certain difficulty in removing microorganisms from the respiratory tree can be listed as factors that facilitate colonization and possible sensitization to $A$. fumigatus. ${ }^{2}$

1. Mestre, Faculdade de Medicina da Universidade de São Paulo (FMUSP), São Paulo, SP, Brasil.

2. Doutor(a), FMUSP, São Paulo, SP, Brasil.

Manuscript received Aug 18 2005, accepted for publication Jan 182006.

Suggested citation: Almeida MB, Bussamra MH, Rodrigues JC. ABPA diagnosis in cystic fibrosis patients: the clinical utility of $\operatorname{lgE}$ specific to recombinant Aspergillus fumigatus allergens. J Pediatr (Rio J). 2006;82: $215-20$.
There is bronchial colonization by $A$. fumigatus in 12 to $57 \%$ of CF patients, ${ }^{3}$ and sensitization to the fungus may vary from 30 to $51 \%$ in all cases. ${ }^{4}$ The prevalence of ABPA varies greatly, from 1 to $15 \%, 5,6$ and increases in line with patient age. ${ }^{7}$ The duration of endobronchial colonization by Pseudomonas aeruginosa and colonization by Stenotrophomonas maltophilia represent risk factors for sensitization to Aspergillus and ABPA, respectively. ${ }^{8}$ Allergic bronchopulmonary aspergillosis is a complicating factor of cystic fibrosis which can result in a devastating combination as lung disease progresses, 9,10 and early diagnosis is of fundamental importance to preventing pulmonary damage and functional deterioration. 11

Despite recent advances in the understanding of ABPA immunopathology, diagnosis in patients with CF is still imprecise. ${ }^{12}$ Even with the adaptation of the classic ABPA diagnostic criteria 13 for use with CF patients, ${ }^{4,14}$ doubt 
very often remains when differentiating patients with ABPA from those who are only sensitized to the fungus.

The development of recombinant components of $A$. fumigatus antigens has made it possible to identify IgE specific to some of these allergens. It has been demonstrated that patients with ABPA react to a larger number of the recombinant allergens in this panel, some of which - $r$ Asp f2, f4 and f6 - are considered specific to ABPA patients. ${ }^{15-18}$ This technique therefore appeared to be very promising for ABPA diagnosis.

The objective of this study was to identify cases of ABPA in a sample of CF patients, employing the technique of detecting IgE specific to the recombinant $A$. fumigatus allergens $r$ Asp f2, f4 and f6 and to compare the results from this method with diagnoses based on the Cystic Fibrosis Foundation (CFF) standardized criteria. ${ }^{14}$

\section{Methods}

This was a cross-sectional study carried out during the routine clinical assessments of patients with diagnoses of $C F$, made in accordance with the CFF consensus statement criteria. ${ }^{19}$ These patients were being treated at the Pediatric Pulmonology Section, Child Institute, at Hospital das Clínicas da Faculdade de Medicina da Universidade de São Paulo (HC-FMUSP) between June 26 and October 10, 2001.

\section{Inclusion criteria}

Patients were recruited if they exhibited any characteristic that, in isolation, could indicate ABPA. Therefore, children and adolescents aged 2 to 20, were included in the presence of any of the following characteristics:

- Personal history of atopic disease with typical manifestations of any of the following diseases: rhinitis, conjunctivitis, dermatitis, angioedema, insect bite allergy, urticaria, food allergy and asthma;

- History of wheezing, even in the absence of asthma;

- The presence of one or more sputum and/or oropharynx cultures positive for Aspergillus during the 2 years prior to enrollment on the study protocol;

- Serum IgE assay result over $500 \mathrm{UI} / \mathrm{ml}$;

- Immediate hypersensitivity skin test positive for $A$. fumigatus;

- Clinical and functional deterioration during the previous 6 months without responding to treatment with antimicrobials.

The clinical and laboratory assessment consisted of: characterization of the type of endobronchial colonization based on the results of previous cultures, Shwachman score, ${ }^{20}$ and spirometry, which was measured only for patients older than 6 years, in accordance with the standards laid down by the American Thoracic Society (ATS), ${ }^{21}$ using a Warren Collins ${ }^{\circledR}$ bell spirometer or a Multispiro ${ }^{\circledR}$ portable spirometer. The Shwachman score is an objective method for evaluating the clinical severity of patients with CF and includes data on physical limitations, physical examination findings, nutritional status and the extent of radiological findings. At our clinic the score is calculated annually for all patients. The tests included in the research protocol are part of the annual laboratory assessments. Annual chest radiography has been substituted by tomography with a volumetric protocol of $10 \mathrm{~mm}$ slices at $10 \mathrm{~mm}$ increments, which results in an effective radiation dose that is much lower than the conventional examination. Immediate hypersensitivity skin testing was performed using the puncture technique, as previously described elsewhere, using standardized extract (International Pharmaceutical Immunology S.A. - IPI, Spain $\left.{ }^{\circledR}\right), 10 \mathrm{mg} / \mathrm{ml}$ histamine positive control and a negative control of phenolated solution at $0.5 \%$ and glycerinated solution. 22 Tests were considered positive if the wheal was $3 \mathrm{~mm}$ or larger, in the presence of a negative control of 0 and a positive control greater than or equal to $3 \mathrm{~mm}$.

Testing (RAST) for serum IgE specific to $A$. fumigatus and for serum IgE specific to the $A$. fumigatus recombinant allergens (ImmunoCAPs) - $r$ Asp f1 (IgERm218), f2 (IgERm219), f3 (IgERm220), f4 (IgERm221) and f6 (IgERm222) - was carried out at the Laboratório Fleury de São Paulo using the automated UniCAP ${ }^{\circledR}$ enzyme immunofluorescence technique in a UniCAP 100 (Pharmacia-Diagnostics) machine. ImmunoCAPs were produced and employed for the titration of allergen specific serum IgE in accordance with specifications described by Crameri et al. ${ }^{23}$ The results were converted into standardized RAST classes from 0 to 4 , in accordance with the manufacturer's instructions (Table 1 ). For data analysis, clinical diagnosis of ABPA was made according to the CFF minimum criteria: ${ }^{14}$ acute or subacute clinical deterioration not attributable to another etiology, total serum IgE concentration of $>500 \mathrm{IU} / \mathrm{mL}(1200 \mathrm{ng} / \mathrm{mL})$, immediate cutaneous reactivity to Aspergillus test wheal of $>3 \mathrm{~mm}$ or demonstration of IgE antibody to $A$. fumigatus, plus at least one of: precipitins to $A$. fumigatus or in vitro demonstration of IgG antibody to $A$. fumigatus, new or recent abnormalities on chest radiography or chest CT with bronchiectasis that did not improve with antibiotics and standard physiotherapy. Precipitin assay is not available at our service and for this reason was not employed.

A diagnosis of ABPA by recombinant antigen specific IgE was defined as assay results greater than or equal to the lower cutoff point for class 3 for IgE antibodies to the A. fumigatus antigens $\mathrm{f} 2, \mathrm{f} 4$ and $\mathrm{f} 6 .{ }^{24}$ Sensitization to $A$. fumigatus was defined as the presence of a positive 
immediate hypersensitivity skin test and/or RAST for $A$. fumigatus and/or serum IgE specific to Asp f1 above $0.35 \mathrm{kU}_{\mathrm{A}} / \mathrm{l}$.

The statistical analysis used Fisher's exact test, the chi-square test or the Mann-Whitney tests to compare groups. Sensitivity and specificity calculations were made using $2 \times 2$ contingency tables.

This project was approved by the medical Research Ethics Committee at the Hospital das Clínicas, Universidade de São Paulo. Patients and their parents or tutors were informed and gave their consent.

Table 1 - Classification of results of allergen specific IgE assay

\begin{tabular}{ccc}
\hline Specific IgE class & $\mathbf{k U}_{\mathbf{A}} / \mathbf{I}$ & Specific IgE level \\
\hline 0 & Below 0.35 & Absent or undetectable \\
1 & $0.35-0.69$ & Low \\
2 & $0.7-3.49$ & Moderate \\
3 & $3.5-17.49$ & High \\
4 & $17.5-49$ & Very high \\
5 & $50-99$ & Very high \\
6 & 100 or more & Very high \\
\hline
\end{tabular}

\section{Results}

Fifty-four CF patients were assessed during the study period and, of these, 39 were considered eligible, although just 32 agreed to participate and to attend for sample collection and testing. The mean age of the study group was 9.8 years $( \pm 3.8)$ and mean Shwachman score was 65 $( \pm 15)$ (Table 2). Eleven patients were identified as sensitized to $A$. fumigatus (34\%). Just three of them had recombinant antigen specific IgE profiles that were compatible with ABPA. Table 3 contains the laboratory data for patients with detectable IgE specific for any of the recombinant antigens or with a diagnosis of ABPA made according to the CFF consensus criteria.

Table 2 - Clinical and laboratory data for the study group

\begin{tabular}{|c|c|c|c|}
\hline Parameter & Mean \pm DP & 95\%CI & Median \\
\hline Age & $9.8 \pm 3.8$ & 8.4-11.2 & 10.1 \\
\hline Score & $65 \pm 15$ & $59.6-70.4$ & 65 \\
\hline $\begin{array}{l}\text { Absolute number } \\
\text { of eosinophils }\end{array}$ & $307 \pm 250$ & 215-398 & 240 \\
\hline Serum IgE & $510 \pm 908$ & $151-869$ & 154 \\
\hline
\end{tabular}

$\mathrm{DP}=$ standard deviation $; 95 \% \mathrm{Cl}=95 \%$ confidence interval for mean .
There was no relationship between the severity of underlying disease, expressed by the Shwachman score, degree of bronchial obstruction or chronic colonization by Pseudomonas aeruginosa with ABPA diagnosed by recombinant antigen specific IgE. A combination of IgE $>500$, positive RAST and skin test positive for $A$. fumigatus had a sensitivity of $75 \%$, specificity of $94 \%$, positive predictive value of $50 \%$ and negative predictive value of 94\% for identifying those ABPA cases diagnosed by recombinant antigen specific IgE.

Three patients were identified as having ABPA according to the CFF criteria. ${ }^{14}$ Two of them had IgE specific to the f2 and f4 fractions and therefore compatible with ABPA. The other patient exhibited only sensitization with IgE specific to the $\mathrm{f} 1$ fraction (Table 3 ).

All patients had CT abnormalities suggestive of bronchiectasis. This examination does not, therefore, differentiate patients by presence or absence of ABPA.

\section{Discussion}

In this population of 32 children and adolescents with CF, two patients with ABPA and 11 individuals sensitized to the fungus were identified by means of skin tests, A. fumigatus specific IgE assay (RAST) and by testing for IgE specific to the recombinant allergens Asp $f 1, f 2, f 3, f 4$ and $f 6$.

It was the difficulty of diagnosing ABPA in CF patients that motivated this study, which was the first opportunity to research $\mathrm{IgE}$ specific to $A$. fumigatus recombinant antigens in our country. The frequency of sensitized individuals was $34 \%$. When the results of the recombinant antigen specific IgE assay were compared with diagnoses made according to the CFF consensus criteria with the benefit of knowledge of the individual clinical histories of each patient, two patients could be identified with a definitive diagnosis of ABPA. There were, therefore, $6.4 \%$ of the sample who had a diagnosis of ABPA.

The prevalence of sensitization to the fungus and of ABPA itself varies across CF treatment centers, ${ }^{4,5}$ and there are no national data on these prevalence rates. Estimates and descriptions of the prevalence of ABPA and of sensitization to $A$. fumigatus based only on classic criteria, even in developed countries, may be underestimated, since the difficulties in diagnosing ABPA in CF are universal. ${ }^{10}$ Cunningham et al. ${ }^{12}$ surveyed the criteria used to diagnose ABPA in specialist clinics in the United Kingdom. Tests such as precipitins, specific IgE and skin tests for $A$. fumigatus, eosinophil count in peripheral blood, total IgE and chest radiography were performed routinely and, on average, the centers performed four of the six tests annually. Nevertheless, there was a large variation in the criteria used to confirm diagnosis in order to start treatment. 
Table 3 - Laboratory data for patients with detectable recombinant antigen specific IgE and/or diagnosis of ABPA by the CFF consensus criteria

\begin{tabular}{cccccccc}
\hline Patient & CFF $*$ & $\begin{array}{c}\text { RAST } \\
\text { Aspergillus } \\
\text { fumigatus }\end{array}$ & $\begin{array}{c}\text { IgE } \\
\text { r Asp f1 }\end{array}$ & $\begin{array}{c}\text { IgE } \\
\text { r Asp f2 }\end{array}$ & $\begin{array}{c}\text { IgE } \\
\text { r Asp f3 }\end{array}$ & $\begin{array}{c}\text { IgE } \\
\text { r Asp f4 }\end{array}$ & $\begin{array}{c}\text { IgE } \\
\text { r Asp f6 }\end{array}$ \\
\hline 1 & no & $12(3)$ & $1.1(2)$ & $\mathbf{3 . 5}(\mathbf{3})$ & $<0.35(0)$ & $2.4(2)$ & $0.6(1)$ \\
2 & ABPA & $29(4)$ & $1.8(2)$ & $\mathbf{1 4 . 4 ( 3 )}$ & $26.4(4)$ & $1.4(2)$ & $<0.35(0)$ \\
3 & ABPA & $39.1(4)$ & $6.8(3)$ & $\mathbf{4 1 . 2 ( 4 )}$ & $3.3(2)$ & $\mathbf{5 ( 3 )}$ & $<0.35(0)$ \\
4 & ABPA & $72.6(5)$ & $0.5(1)$ & $<0.35(0)$ & $<0.35(0)$ & $<0.35(0)$ & $<0.35(0)$ \\
5 & no & $7.6(3)$ & $3.6(3)$ & $2.9(2)$ & $<0.35(0)$ & $<0.35(0)$ & $0.4(1)$ \\
6 & no & $<0.35(0)$ & $6.2(3)$ & $<0.35(0)$ & $<0.35(0)$ & $0.9(1)$ & $<0.35(0)$ \\
\hline
\end{tabular}

ABPA = allergic bronchopulmonary aspergillosis; CFF = Cystic Fibrosis Foundation; RAST = IgE specific to A. fumigatus.

* Diagnosis of ABPA by the CFF. ${ }^{14}$ Precipitin assay was not used.

$\lg$ E assay expressed in $\mathrm{kU}_{\mathrm{A}} / \mathrm{l}$ (class). Laboratory results in bold are compatible with $\mathrm{ABPA}$.

Patients 2 and 3 were defined as having ABPA and were treated. Patient 1 was defined as a carrier of previous ABPA ("serological scar").

Prevalence data therefore depends on which criteria and laboratory tests are considered when defining sensitization and ABPA. ${ }^{14}$ It is also known that immunological test results can fluctuate over time, with intra-individual variation. ${ }^{25}$ Additionally, there are reports of patients who had presented sensitization and then ceased to have test results compatible with sensitization during follow-up. 26

In this sample, the association of positive skin test for A. fumigatus hypersensitivity, absolute number of eosinophils in peripheral blood over $500 / \mathrm{mm}^{3}$, serum IgE assay result over $500 \mathrm{UI} / \mathrm{ml}$ and positive RAST for $A$. fumigatus, offered good specificity for ABPA diagnosis. In addition to the importance of these tests for periodic screening of patients at risk from ABPA, the combination is useful because these tests are simpler and more likely to be available than the test for $A$. fumigatus recombinant antigen specific IgE.

In patients with $C F$, the relationship between high serum IgE and ABPA has already been reported by several authors, and this is one of the laboratory variables included in the classic diagnostic criteria. 5,27 What is peculiar to CF patients is that these IgE levels may not be so high, 28,29 as the CFF consensus emphasizes in suggesting that annual ABPA screening be performed if there is a strong clinical suspicion even when IgE $<500 \mathrm{UI} / \mathrm{ml}^{14}$ The level of serum IgE varies according to the phase of disease activity, ${ }^{1}$ and reduced levels after treatment with corticosteroids is another factor to be taken into account when diagnosing ABPA. 4,5
Banerjee et al. ${ }^{17}$ demonstrated that CF patients with ABPA exhibit significantly higher levels of IgE specific to the Asp f2 antigen, when compared with controls, with $100 \%$ specificity. This antigen appears to be involved with the adherence of the fungus to the extracellular matrix. The native and recombinant forms of this antigen were assessed and considered immunologically comparable and useful for specific diagnosis of ABPA. In ABPA fungus grows in the airways and the immune reaction that follows results in the destruction of these microorganisms, with exposure of intracellular antigens. It is believed that detecting IgE specific to these intracellular antigens of Aspergillus indicates the disease. In contrast, immunoresponse against proteins secreted after germination of spores, such as $r$ Asp $f 1$ and $r$ Asp f3, is observed both in individuals with ABPA and in those who are sensitized. Preliminary studies of the cellular distribution of the $r$ Asp $f 4$ antigen indicate that it is a secreted protein. ${ }^{30}$ The $r$ Asp $f 6$ antigen has been identified as a protein specific to the hyphae of Aspergillus, and these hyphae are only present in ABPA. ${ }^{31}$ Therefore, identification of IgE specific to the $r$ Asp $f 4$ and $r$ Asp $f 6$ antigens can also be used as a marker specific to ABPA. ${ }^{30}$ In our sample, both ABPA patients' assay results for IgE specific to $r$ Asp f6 were defined as class $I$, in common with what has been reported by other researchers who observed lower $r$ Asp f6 specific IgE levels, when compared with the other antigens. ${ }^{15,30,32}$

When the ABPA diagnosis made by assaying IgE specific to recombinant antigens was compared with the 
diagnosis made by the CFF criteria, there was only agreement on two patients, and therapeutic intervention was indicated for them. After analyzing the clinical progress of the two patients who had been diagnosed by one method, but not by the other, we concluded that:

- Patient 1, with a profile of IgE for recombinant antigens suggestive of ABPA and a previous history of treated ABPA, was considered a probable case of a serological scar, which was also described in an epidemiological study by Geller. ${ }^{4}$ When the profiles of IgE to recombinant antigens are assessed individually, at different points of the clinical course, IgE specific to $r$ Asp f3 appears to remain high during remission; ${ }^{24,33}$ although this behavior was not observed in our patient.

- Patient 4 fulfilled the CFF consensus criteria for a diagnosis of ABPA, but did not have a profile of IgE for recombinant antigens compatible with the disease. Laboratory data only suggested sensitization to the fungus. This is an understandable situation because of the overlapping of the clinical signs of the two diseases, in a severe case of $\mathrm{CF}$, with advanced lung disease and total serum $\mathrm{IgE}=4,353 \mathrm{UI} / \mathrm{ml}$.

Cutoff values have not yet been defined for these assays of IgE for recombinant antigens, but published studies all adopt values compatible with a minimum of class III (over $3.5 \mathrm{kU}_{\mathrm{A}} / \mathrm{l}$ ). ${ }^{24}$ Knutsen et al., ${ }^{33}$ studied 15 patients with ABPA - 23 sensitized and 19 CF controls demonstrating that IgE specific to the 44 fraction has the greatest sensitivity and specificity for detecting ABPA. Patient 6 presented detectable levels of IgE to $r$ Asp $f 4$, but at a low level (class 1 ), and patient 5 had classes I and II IgE specific for $r$ Asp $f 6$ and $f 2$, respectively, both without clinical status of ABPA. These patients may represent an initial form of ABPA, with minimal abnormalities mainly in laboratory test results. These patients are undergoing clinical surveillance and annual laboratory screening due to the possibility of ABPA in the future.

Casaulta et al. ${ }^{24}$ performed a longitudinal analysis, over approximately 3 years, of the clinical and laboratory data of 23 patients with ABPA, comparing them with Aspergillus sensitized and unsensitized controls. These patients presented high levels of IgE specific to $r$ Asp $f 4$ and $r$ Asp f6, and an association with total serum IgE over $1,000 \mathrm{UI} / \mathrm{ml}$ allowed ABPA to be confirmed with high specificity (100\%) and sensitivity (64\%). Under this treatment, specific and total IgE levels were reduced but did not normalize, and pulmonary function remained stable despite the treatment. Testing for IgE to recombinant antigens of $A$. fumigatus was considered useful for identifying patients at increased risk of ABPA, but laboratory data was not sufficient to monitor treatment.

Allergic bronchopulmonary aspergillosis is an infrequent complication of $\mathrm{CF}$ and diagnostic confirmation remains a challenge. Despite the efforts directed at establishing diagnostic criteria adapted to CF patients, diagnostic failures still occur. We performed a systematic investigation comparing two distinct strategies, the CFF criteria and the detection of IgE specific to recombinant antigens, and found that the diagnostic conclusions were discordant. Assaying IgE to recombinant antigens of $A$. fumigatus appears to us to be a promising technique for the early detection of sensitization to the fungus and of ABPA itself, perhaps during a phase with little clinical pulmonary symptomology. However, the true utility of this laboratory method to the clinical practice has not yet been defined. Despite the high specificity previously described for this laboratory method, neither diagnostic confirmation nor the treatment can be divorced from the clinical condition of the patients. Further longitudinal studies with larger numbers of patients remain necessary to achieve diagnosis during the diverse stages of the disease and for detecting relapses and establishing cure criteria after therapeutic intervention.

\section{Acknowledgements}

The authors are grateful for the help of Dr. Mario Camargo, researcher at the Laboratório Fleury for performing the diagnostic tests, and Dr. Sônia Regina Testa da Silva Ramos for interpreting the statistical analysis.

\section{References}

1. Skov M, Poulsen LK, Koch C. Increased antigen-specific Th-2 response in allergic bronchopulmonary aspergillosis (ABPA) in patients with cystic fibrosis. Pediatr Pulmonol. 1999;27:74-9.

2. Zeaske R, Bruns WT, Fink JN, Greenberger PA, Colby H, Liotta $\mathrm{JL}$, et al. Immune responses to Aspergillus in cystic fibrosis. J Allergy Clin Immunol. 1988;82:73-7.

3. Milla CE, Wielinski CL, Regelmann WE. Clinical significance of the recovery of Aspergillus species from the respiratory secretions of cystic fibrosis patients. Pediatr Pulmonol. 1996;21:6-10.

4. Geller DE, Kaplowitz $H$, Light MJ, Colin AA. Allergic bronchopulmonary aspergillosis in cystic fibrosis: reported prevalence, regional distribution, and patient characteristics. Scientific Advisory Group, Investigators, and Coordinators of the Epidemiologic Study of Cystic Fibrosis. Chest. 1999;116: 639-46.

5. Mastella G, Rainisio M, Harms HK, Hodson ME, Koch C, Navarro $\mathrm{J}$, et al. Allergic bronchopulmonary aspergillosis in cystic fibrosis. A European epidemiological study. Epidemiologic Registry of Cystic Fibrosis. Eur Respir J. 2000;16:464-71.

6. Zander DS. Allergic bronchopulmonary aspergillosis: an overview. Arch Pathol Lab Med. 2005;129:924-8.

7. Nelson LA, Callerame ML, Schwartz RH. Aspergillosis and atopy in cystic fibrosis. Am Rev Respir Dis. 1979;120:863-73.

8. Ritz N, Ammann RA, Casaulta Aebischer C, Schoeni-Affolter F, Schoeni MH. Risk factors for allergic bronchopulmonary aspergillosis and sensitisation to Aspergillus fumigatus in patients with cystic fibrosis. Eur J Pediatr. 2005;164:577-82. Epub 2005 May 31.

9. Milla CE. Allergic bronchopulmonary aspergillosis and cystic fibrosis. Pediatr Pulmonol. 1999;27:71-3.

10. Slavin RG. ABPA in CF: a devastating combination. Pediatr Pulmonol. 1996;21:1-2 
11. Kumar R. Mild, moderate, and severe forms of allergic bronchopulmonary aspergillosis: a clinical and serologic evaluation. Chest. 2003;124:890-2.

12. Cunningham S, Madge SL, Dinwiddie R. Survey of criteria used to diagnose allergic bronchopulmonary aspergillosis in cystic fibrosis. Arch Dis Child. 2001;84:89.

13. Rosenberg $M$, Patterson R, Mintzer R, Cooper $B J$, Roberts $M$, Harris KE. Clinical and immunologic criteria for the diagnosis of allergic bronchopulmonary aspergillosis. Ann Intern Med. 1977;86:405-14.

14. Stevens DA, Moss RB, Kurup VP, Knutsen AP, Greenberger P, Judson $M$, et al. Allergic bronchopulmonary aspergillosis in cystic fibrosis - state of the art: Cystic Fibrosis Foundation Consensus Conference. Clin Infect Dis. 2003;37 Suppl 3:S225-64.

15. Kurup VP, Banerjee B, Hemmann S, Greenberger PA, Blaser K, Crameri R. Selected recombinant Aspergillus fumigatus allergens bind specifically to IgE in ABPA. Clin Exp Allergy. 2000;30:988-93.

16. Crameri R, Hemmann S, Ismail C, Menz G, Blaser K. Diseasespecific recombinant allergens for the diagnosis of allergic bronchopulmonary aspergillosis. Int Immunol. 1998;10:1211-6.

17. Banerjee B, Greenberger PA, Fink JN, Kurup VP. Immunological characterization of Asp f 2, a major allergen from Aspergillus fumigatus associated with allergic bronchopulmonary aspergillosis. Infect Immun. 1998;66:5175-82.

18. Nikolaizik WH, Moser M, Crameri R, Little S, Warner JO, Blaser $\mathrm{K}$, et al. Identification of allergic bronchopulmonary aspergillosis in cystic fibrosis patients by recombinant Aspergillus fumigatus I/a-specific serology. Am J Respir Crit Care Med. 1995;152:634-9.

19. Rosenstein BJ, Cutting GR. The diagnosis of cystic fibrosis: a consensus statement. Cystic Fibrosis Foundation Consensus Panel. J Pediatr. 1998;132:589-95.

20. Shwachman $H$, Kulczycki LL. Long-term study of one hundred five patients with cystic fibrosis; studies made over a five- to fourteen-year period. Am J Dis Child. 1958;96:6-15.

21. Standardization of spirometry, 1994 Update. American Thoracic Society. Am J Respir Crit Care Med. 1995;152:1107-36.

22. Bousquet J, Van Cauwenberge P, Khaltaev N; Aria Workshop Group; World Health Organization. Allergic rhinitis and its impact on asthma. J Allergy Clin Immunol. 2001;108:S147-334.

23. Crameri R, Lidholm J, Gronlund H, Stuber D, Blaser K, Menz G. Automated specific IgE assay with recombinant allergens: evaluation of the recombinant Aspergillus fumigatus allergen I in the Pharmacia Cap System. Clin Exp Allergy. 1996;26:1411-9.
24. Casaulta C, Fluckiger S, Crameri R, Blaser K, Schoeni MH. Time course of antibody response to recombinant Aspergillus fumigatus antigens in cystic fibrosis with and without ABPA. Pediatr Allergy Immunol. 2005;16:217-25.

25. Hutcheson PS, Rejent AJ, Slavin RG. Variability in parameters of allergic bronchopulmonary aspergillosis in patients with cystic fibrosis. J Allergy Clin Immunol. 1991;88:390-4.

26. Hutcheson PS, Knutsen AP, Rejent AJ, Slavin RG. A 12-year longitudinal study of Aspergillus sensitivity in patients with cystic fibrosis. Chest. 1996;110:363-6.

27. Laufer $P$, Fink JN, Bruns WT, Unger GF, Kalbfleisch JH, Greenberger PA, et al. Allergic bronchopulmonary aspergillosis in cystic fibrosis. J Allergy Clin Immunol. 1984;73:44-8.

28. Zeaske R, Bruns WT, Fink JN, Greenberger PA, Colby H, Liotta $\mathrm{J} L$, et al. Immune responses to Aspergillus in cystic fibrosis. J Allergy Clin Immunol. 1988;82:73-7.

29. Maiz L, Cuevas M, Quirce S, Pacheco A, Escobar H. Allergic bronchopulmonary aspergillosis with low serum IgE levels in a child with cystic fibrosis. J Allergy Clin Immunol. 1997;100:431-2.

30. Crameri R, Hemmann S, Ismail C, Menz G, Blaser K. Diseasespecific recombinant allergens for the diagnosis of allergic bronchopulmonary aspergillosis. Int Immunol. 1998; 10:1211-6.

31. Schwienbacher M, Israel L, Heesemann J, Ebel F. Asp f6, an Aspergillus allergen specifically recognized by IgE from patients with allergic bronchopulmonary aspergillosis, is differentially expressed during germination. Allergy. 2005;60:1430-5.

32. Hemmann S, Nikolaizik WH, Schoni MH, Blaser K, Crameri R. Differential IgE recognition of recombinant Aspergillus fumigatus allergens by cystic fibrosis patients with allergic bronchopulmonary aspergillosis or Aspergillus allergy. Eur J Immunol. 1998;28:1155-60.

33. Knutsen AP, Hutcheson PS, Slavin RG, Kurup VP. IgE antibody to Aspergillus fumigatus recombinant allergens in cystic fibrosis patients with allergic bronchopulmonary aspergillosis. Allergy. 2004;59:198-203.

Correspondence:

Marina Buarque de Almeida

Rua Martim Francisco, 748 casa 2

CEP 01226-000 - São Paulo, SP - Brazil

Tel.: + 55 (11) 3666.4678

Fax: + 55 (11) 3032.5226

E-mail: m.buarque@terra.com.br 